\title{
A static video summarization method based on the sparse coding of features and representativeness of frames
}

\author{
Dong-ju Jeong ${ }^{1}$, Hyoung Jin $\mathrm{Yoo}^{2}$ and Nam Ik Cho ${ }^{1 *}$
}

\begin{abstract}
This paper presents a video summarization method that is specifically for the static summary of consumer videos. Considering that the consumer videos usually have unclear shot boundaries and many low-quality or meaningless frames, we propose a two-step approach where the first step skims a video and the second step performs content-aware clustering with keyframe selection. Specifically, the first step removes most of redundant frames that contain only little new information by employing the spectral clustering method with color histogram features. As a result, we obtain a condensed video that is shorter and has clearer temporal boundaries than the original. In the second step, we perform rough temporal segmentation and then apply refined clustering for each of the temporal segments, where each frame is represented by the sparse coding of SIFT features. The keyframe selection from each cluster is based on the measure of representativeness and visual quality of frames, where the representativeness is defined from the sparse coding and the visual quality is the combination of contrast, blur, and image skew measures. The problem of keyframe selection is to find the frames that have both representativeness and high quality, which is formulated as an optimization problem. Experiments on videos with various lengths show that the resulting summaries closely follow the important contents of videos.
\end{abstract}

Keywords: Video summarization, Consumer video, Video analysis, Keyframe extraction, Keyframe selection

\section{Introduction}

The wide spread use of mobile phones and wearable cameras has brought a culture that many people enjoy capturing and sharing images and videos with one another. It is especially notable that users are capable of storing and accessing more and larger videos than before. Hence, it is also becoming important to efficiently search for the videos that contain the desired contents, where video summaries can help them to efficiently catch the main points and look for relevant data [1].

Video summarization is the condensation of a video into a storyboard that contains keyframes or several video segments [2], the goal of which is to make the video as small as possible while keeping the flow of the story and essential parts of frames and/or video clips. There have

*Correspondence: nicho@snu.ac.kr

1 Department of Electrical and Computer Engineering, INMC, Seoul National University, 1 Gwanak-ro, Gwanak-gu, Seoul 08826, Korea

Full list of author information is available at the end of the article been a huge number of methods for the summarization of well-edited videos such as movies and TV contents [3-6], where a well-edited video means that it has clear shot boundaries and was shot under well-controlled environments with stable and high-quality cameras. In contrast to these well-edited videos, consumer videos (shot by hand-held mobile cameras, camcorders, or egocentric cameras such as sports glasses and action cams) usually have unclear or no shot boundaries and also contain many low-quality frames or even meaningless ones. Hence, above mentioned video summarization methods, which were designed for the well-edited videos, do not work well for consumer videos, so recent studies are targeting consumer videos $[2,7-9]$.

In general, there are some challenges related to the consumer video summarization. First, it should work for various lengths of shots; especially it has to be able to treat a shot that may be very long in duration. This also means that it does not have to focus too much on visual 
details in a frame and should avoid using a model that cannot represent the whole visual contents of a long video. Second, for treating various kinds of videos, it is desired to use features that can play important roles in any type of video. One may assume that the frames containing a person's face or moving objects are important, but this is not always the case. The third one is that in addressing the keyframe selection problem, we are given the difficult choice between two things: choosing representative frames or quality ones. Last, because the desired number of keyframes is itself subjective, a video summarization method should be able to adjust the number of keyframes.

Considering the challenges stated above, we propose a new algorithm that is especially suited for summarizing long consumer videos in reasonable computation times. The proposed algorithm consists of two steps: the first step is skimming a video, i.e., reducing the number of frames by using spectral clustering with simple color histograms, which greatly reduces time for the refined clustering and keyframe selection in the second step. The refined clustering in the second step is to cluster the images based on the sparse coding of SIFT features. In this process, we can also define the representativeness with the sparse coding coefficients. We also formulate the quality measures of each frame in this step such as contrast, blur, and image skew. The main problem is to find the frames that have both representativeness and high quality, which is formulated as an optimization problem in the form of graph matching $[10,11]$. Also, since not every method can meet the various requirements of users or diverse kinds of videos, the algorithm is designed to easily adopt new features if needed. Figure 1 shows a flowchart of our method, where each block contains notations for showing what is obtained from each processing step. Many experimental video clips and their step-by-step results produced by our algorithm are available in http://ispl.snu.ac.kr/ jeongdj/ videosummary.html.

The rest of this paper is organized as follows: The second section introduces the related works on video summarization, the third section presents video skimming and temporal segmentation, and the fourth section describes measuring the quality of frames and selecting keyframes. The experimental results and the conclusion are presented in the last two sections.

\section{Related works}

The conventional algorithms for video summary were developed for videos that are manipulated by professional editors. These well-edited contents have mostly clear shot boundaries but sometimes ambiguous ones such as fade in/out and camera view changes. Hence, the conventional algorithms focused on finding the ambiguous shot boundaries and also finding the keyframes in a shot that represent the shot. For example, Omidyeganeh et al. [3] developed a keyframe extraction algorithm, where they exploited features extracted from the wavelet transform subbands of each frame to partition the whole video into subshots and select the final keyframes. Chasanis et al. [4] proposed an algorithm that uses shot boundary detection and the spectral clustering method, and they formulated the scene detec-

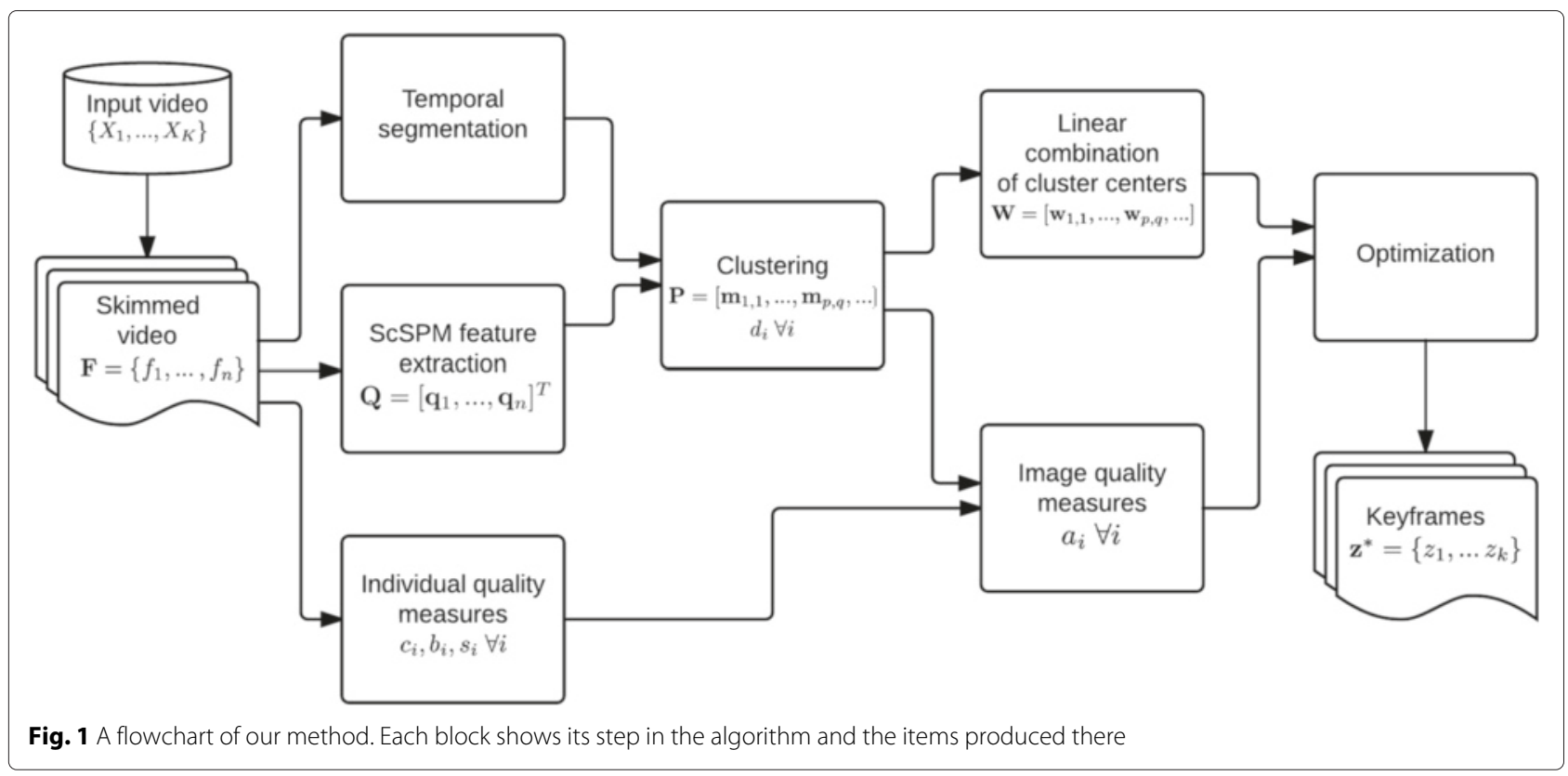


tion as keyframe alignment. Although there are also many methods that do not detect shot boundaries [5, $12,13]$, dividing the whole video into subshots contributes to stabilize the keyframe extraction results [3, 4, 14-17]. Given its importance, shot boundary detection is still a much studied topic [18-20].

However, as stated in the introduction, the above stated methods do not work well for unstructured videos (amateur and consumer videos) because they are poorly shot and edited. These videos have many unclear shot boundaries, and they also have meaningless, redundant, or lowquality frames. Hence, studies focusing on unstructured videos have recently been gaining attention, which are focused on static or dynamic summaries of consumer and/or egocentric videos. For some examples of static summarization methods that we study in this paper, Cong et al. [7] considered video summarization as a dictionary selection problem, where keyframes that represent the whole video content form the dictionary. But this method is not suitable for long and complex videos because the long content is not well represented by a dictionary. The algorithm proposed by Ejaz et al. [8] was based on the assumption that visual attention would indicate the importance of each frame in general videos. They used spatial and temporal attention values and emphasized the motion information. However, this framework is vulnerable to some factors such as the capturers' or objects' movement and quality of frames and depends on how much time one determines to make a scene. Meanwhile, there have been some algorithms targeting only egocentric videos $[2,9]$. They are similar to those for general consumer videos in that they perform temporal segmentation and score the frames based on certain criteria. However, they make use of information that is confined to the first-person views and needs high computatonal loads, which makes them unsuitable for videos not captured by first-person cameras and/or long videos. There are also several methods that focus on temporal segmentation of consumer videos $[1,9,21,22]$, where "temporal segmentation" may work as "shot boundary detection" for welledited videos and it is also applied to video annotation algorithms [23, 24].

\section{Video skimming and temporal segmentation}

Consumer videos usually have redundant frames, when compared with movie or TV contents that are well edited. Hence, we first skim the video, i.e., remove the redundant frames by using spectral clustering with simple color histograms. The skimmed video is then temporally segmented for the second step.

\subsection{Video skimming by spectral clustering}

To remove the redundant frames very fast, we first apply a clustering method with simple features. Then, from each of the clusters, we find some frames that are nearest to the center of the cluster. This results in a skimmed video, where redundant frames are naturally reduced and the scenes are represented by a relatively small number of frames, and also most of the transient scenes are eliminated.

To be specific, the video is first divided into evenly spaced subshots $\mathbf{X}_{1}, \ldots, \mathbf{X}_{K}$, each of which is about 3-4 min long in our implementation. Note that the first step is to roughly skim the video, and that the length of even spacing is not so important but needs to be short enough not to miss some important frames. For each subshot, we apply spectral clustering [25] with the normalized HSV histogram as a feature, for the fast grouping of similar frames as in [4]. Also, according to the report in [26] that the Hellinger distance measure instead of the Euclidean distance gives more plausible image retrieval results, we also apply this measure for the comparison of histogram features. Specifically, we $L 1$ normalize the histograms and then square root each element so that each of them has unit $L 2$ norm.

To be more precise with the spectral clustering of each subshot defined above, let us denote the $k$ th subshot as $\mathbf{X}_{k}=\left[x_{1}, x_{2}, x_{3}, \ldots, x_{N_{k}}\right], k \in\{1, \ldots, K\}$ where $K$ is the number of subshots and $N_{k}$ is the size of $\mathbf{X}_{k}$. For each subshot, the similarity matrix $\mathbf{A} \in \mathbb{R}^{N_{k} \times N_{k}}$ and the matrix $\mathbf{L}=$ $\mathbf{D}^{-1 / 2} \mathbf{A D}^{-1 / 2}$ are computed, where $\mathbf{D}$ is the degree matrix each of whose diagonal entries is the sum of the elements belonging to the same row. The matrix $\mathbf{L}$ is used as a proxy of the Laplacian matrix I - L [25], where I is the identity matrix. Then, the spectral clustering to divide the $k$ th subshot into $M$ clusters is to solve a relaxed optimization problem:

$$
\max _{\mathbf{Y}} \operatorname{trace}\left(\mathbf{Y}^{T} \mathbf{L Y}\right) \quad \text { s.t. } \quad \mathbf{Y}^{T} \mathbf{Y}=\mathbf{I}_{M}
$$

where $\mathbf{Y}$ is the relaxed version of $\mathbf{Z} \in \mathbb{R}^{N_{k} \times M}$ defined as

$$
Z(i, j)=\left\{\begin{array}{l}
1 \text { if } x_{i} \in(j \text { th cluster }) \\
0 \text { otherwise. }
\end{array}\right.
$$

It is known that the maximum value of $\operatorname{trace}\left(\mathbf{Y}^{T} \mathbf{L Y}\right)$ in (1) is equal to the sum of the $M$ largest eigenvalues of $\mathbf{L}$ [27]. ${ }^{1}$ Thus, in our clustering process, the number of clusters is determined based on the eigenvalues of the Laplacian matrix defined for the spectral clustering, i.e., as the number of eigenvalues that are larger than a threshold $\theta_{a}$. For more details about spectral clustering, refer to $[4,27,28]$. From each of the clusters obtained by the above process, we extract several frames that are close to the center of the cluster where the number of extracted frames is defined to be proportional to the cluster size. The extracted frames are ordered chronologically, which is referred to as the skimmed video in this paper. With the appropriate threshold $\theta_{a}$ (which will be specified in the experiment section), we obtain a 
decent number of frames for each cluster that contains many similar frames and sometimes rare and transient frames. Thus, it is possible to remove the redundant frames and leave only a small number of frames by selecting only the ones that are close to the cluster centers. The results of the first step, i.e., the skimmed videos for the input videos, are also available at the above stated site. For the rest of this paper, the "frames" refer to the frames in this skimmed video, which are denoted as $\mathbf{F}=\left\{f_{1}, \ldots, f_{n}\right\}$.

\subsection{Temporal segmentation}

The clustering in the above procedure is somewhat rough in that we use small $\theta_{a}$ that produces many frames similar to the cluster centers, and also that the neighboring clusters contain almost the same results because the video is first evenly segmented so that neighboring segments may contain the same scene. The purpose of this rough clustering is just to obtain a skimmed video and also to remove redundant or unimportant frames for reducing computations for the following keyframe selection steps. For the keyframe selection, we need to apply refined clustering to the skimmed video and then find keyframes from each cluster. Temporal segmentation provides some video segments in each of which the clustering is performed. In this way, we force each segment to produce one or more keyframes because each segment is sufficiently large and important enough to have a keyframe. Without the temporal segmentation step, the clustering would be performed with all the frames in $\mathbf{F}$, and some frame representing an important part of the video might be precluded from the video summary.

For the temporal segmentation, we perform the kernel temporal segmentation (KTS) method [21], which detects the change points of a signal (a video stream here) statistically. Since any kind of features can be used for this method, we reuse the color histograms that were used in the above process for saving the computations. Let $\mathbf{H}=\left[\boldsymbol{\pi}\left(f_{1}\right), \ldots, \boldsymbol{\pi}\left(f_{n}\right)\right]$ denote the color histograms of the frames. We define a kernel function $K: \mathbf{H} \times \mathbf{H} \rightarrow \mathbb{R}$ as

$$
\begin{aligned}
K\left(\boldsymbol{\pi}\left(f_{i}\right), \boldsymbol{\pi}\left(f_{j}\right)\right) & =\boldsymbol{\Phi}\left(f_{i}\right) \cdot \boldsymbol{\Phi}\left(f_{j}\right) \\
& =1-\frac{1}{\sqrt{2}}\left\|\boldsymbol{\pi}\left(f_{i}\right)-\boldsymbol{\pi}\left(f_{j}\right)\right\|_{2}
\end{aligned}
$$

where $\boldsymbol{\Phi}: \mathbf{H} \rightarrow \mathcal{H}$ is the feature mapping indirectly defined by $K$, and $\mathcal{H}$ is the associated feature space. The KTS algorithm performs segmentation by minimizing an objective function such that the sum of withinsegment variances is minimized, while penalizing oversegmentation simultaneously:

$$
\min _{m ; t_{1}, \ldots, t_{m}} J_{m, n}:=L_{m, n}+C \cdot g(m, n)
$$

where $m$ is the number of segment boundaries at $t_{1}, \ldots, t_{m}$, and $L_{m, n}$ is defined as

$$
\begin{aligned}
& L_{m, n}=\sum_{i=0}^{m} v_{t_{i}, t_{i+1}}, v_{t_{i}, t_{i+1}}=\sum_{t=t_{i}}^{t_{i+1}-1}\left\|\boldsymbol{\Phi}\left(f_{t}\right)-\boldsymbol{\mu}_{i}\right\|_{2}^{2}, \\
& \boldsymbol{\mu}_{i}=\frac{\sum_{t=t_{i}}^{t_{i+1}-1} \boldsymbol{\Phi}\left(f_{t}\right)}{t_{i+1}-t_{i}}, t_{0}=1, t_{m+1}=n+1,
\end{aligned}
$$

and $g(m, n)$ can be computed as

$$
g(m, n)=m(\log (n / m)+1)
$$

Note that $L_{m, n}$ is the sum of all the within-segment variances and $g(m, n)$ increases when we add more segments, which penalizes over-segmentation. The constant $C$ controls the trade-off between under-and over-segmentation. The within-segment variances in (5) can be computed as

$$
\begin{aligned}
v_{t_{i}, t_{i+1}} & =\sum_{t=t_{i}}^{t_{i+1}-1}\left\|\boldsymbol{\Phi}\left(f_{t}\right)\right\|_{2}^{2}-2 \sum_{t=t_{i}}^{t_{i+1}-1} \boldsymbol{\Phi}\left(f_{t}\right) \cdot \boldsymbol{\mu}_{i}+\left(t_{i+1}-t_{i}\right)\left\|\boldsymbol{\mu}_{i}\right\|_{2}^{2} \\
& =\sum_{t=t_{i}}^{t_{i+1}-1}\left\|\boldsymbol{\Phi}\left(f_{t}\right)\right\|_{2}^{2}-\frac{1}{t_{i+1}-t_{i}}\left\|\sum_{t=t_{i}}^{t_{i+1}-1} \boldsymbol{\Phi}\left(f_{t}\right)\right\|_{2}^{2} \\
& =\sum_{t=t_{i}}^{t_{i+1}-1} K\left(\pi\left(f_{t}\right), \boldsymbol{\pi}\left(f_{t}\right)\right)-\frac{1}{t_{i+1}-t_{i}} \sum_{s=t_{i}}^{t_{i+1}-1} \sum_{t=t_{i}}^{t_{i+1}-1} K\left(\pi\left(f_{s}\right), \boldsymbol{\pi}\left(f_{t}\right)\right) .
\end{aligned}
$$

Since nothing but the inner products between $\boldsymbol{\Phi}$ 's needs to be known, our own kernel function and the Gram matrix can be efficiently exploited in the algorithm. Optimization of the objective function is based on dynamic programming, and it was reported in [21] that the time complexity of the algorithm is $\mathcal{O}\left(m_{\max } n^{2}\right)$ where $m_{\max }$ is the largest possible number of segment boundaries. Also, the $n \times n$ Gram matrix and its cumulative sums are calculated for the within-segment variances, and the amount of memory they take up is one of the major problems. Because the runtime and memory complexity increase quadratically with $n$, reduction of redundant frames (decrease in $n$ ) in the above process is very effective. For example, a 3-h-long video is reduced to 739 frames in the video skimming step, and the temporal segmentation and the next procedures are performed with $n=739$.

\section{Image quality measure and keyframe extraction}

A keyframe is an image frame that represents a shot very well. In addition to this, in the case of a consumer video, we need to consider the quality of frames because there are many low-quality frames in consumer videos due to uncontrolled illumination, shaking, skewed shots, out-of-focus blur, etc. Hence, we balance the measure of 
representativeness and quality of a frame when selecting keyframes.

Specifically, our task of keyframe extraction is to find the optimal keyframe set $\mathbf{z}^{*}=\left\{z_{1}, \ldots, z_{k}\right\}$ from the entire candidate frame set $\mathbf{F}$, where $k$ is the number of keyframes, according to an optimization criterion:

$$
\mathbf{z}^{*}=\underset{\mathbf{z} \subset \mathbf{F}}{\operatorname{argmin}} \lambda E_{1}(\mathbf{z})+E_{2}(\mathbf{z})
$$

where $E_{1}$ and $E_{2}$ are the cost functions related to frame quality and representativeness, respectively. To solve this problem, we formulate our task as a graph matching problem, which can be effectively used to consider both frame quality and representativeness.

\subsection{Measuring the quality and representativeness of a frame}

In order to give a narrow chance of being a keyframe to the low-quality images, we measure the contrast as variance of pixels. The metric of blurriness is measured according to [29] and the skewness from [30]. For encoding these measures into the cost function $E_{1}$, we denote the badness of contrast, blur, and skewness of the $i$ th frame as $c_{i}, b_{i}$, and $s_{i}$, respectively, which are normalized into the range of $[0,1]$ by the sigmoid function (a larger value means worse quality):

$$
\begin{aligned}
& c_{i}=2 /\left(1+\exp \left(-\tau_{1} \cdot c_{i}^{\prime}\right)\right), \quad b_{i}=2 /\left(1+\exp \left(-\tau_{2} \cdot b_{i}^{\prime}\right)\right), \\
& s_{i}=\left\{\begin{array}{ll}
\tau_{3} \cdot s_{i}^{\prime} & \text { if } \tau_{3} \cdot s_{i}^{\prime}<1 \\
1 & \text { otherwise }
\end{array} \quad \forall i \in\{1, \ldots, n\}\right.
\end{aligned}
$$

where $c_{i}^{\prime}, b_{i}^{\prime}$, and $s_{i}^{\prime}$ are the original measures of $c_{i}, b_{i}$, and $s_{i}$, respectively. Because $c_{i}^{\prime} \in[0,0.25]$ with normalized intensities and $b_{i}^{\prime} \in[0,1]$ as in [29], we choose different parameters $\tau_{1}=-20$ and $\tau_{2}=-5$ for the sigmoid functions. Also, since the value of $s_{i}^{\prime}$ means the degree of skewness in radians, we set $s_{i}$ to be proportional to $s_{i}^{\prime}$ with $\tau_{3}=2$. These types of metrics can be used for the summarization of any type of video and the quality measures may also be replaced with other metrics according to some specific input video types.

In addition, we observe that the users try to keep the cameras still (in the case of consumer video shots) or keep the sight for a while (in the case of egocentric video) when they are taking important scenes or observing prominent objects. Hence, in the consumer videos, the shots from still camera works are usually important ones, and also the image qualities are good due to stillness of a camera. Hence, we define one more measure of image quality for this, as the distance of a frame from its cluster center. Specifically, since the frames have small differences in the still scenes, the distance of the $i$ th frame from its cluster center, denoted as $d_{i}$, is used as an additional measure of image quality.

For this, we apply more refined clustering to the temporal segments obtained in the above procedure and find the distance of each frame from its cluster center. We refer "refined clustering" as the one using the SIFT vectors, rather than the simple color histograms that were used in the first step, for considering the patterns of objects in the scene and alleviating the demerits of color features. More specifically, we adopt the idea of spatial pyramid matching with dense SIFT descriptors (ScSPM) [31] devised for image classification, because we may regard the clustering as an image classification task and the ScSPM vector is a kind of feature that can overcome the limitation of color features; they do not distinguish some scenes with similar color distributions [3]. The ScSPM method is based on sparse representation, but $l_{2}$ regularizer can also be used with a sufficiently over-complete dictionary according to the studies of collaborative representation [32, 33]. That is, if we denote $\mathbf{y}, \mathbf{x}^{*}$, and $\mathbf{D}$ as a SIFT descriptor, its coefficient vector, and a visual dictionary respectively, then $\mathbf{x}^{*}$ is given by

$$
\begin{aligned}
\mathbf{x}^{*} & =\underset{\mathbf{x}}{\operatorname{argmin}}\|\mathbf{y}-\mathbf{D} \mathbf{x}\|_{2}^{2}+\alpha\|\mathbf{x}\|_{2}^{2} \\
& =\left(\mathbf{D}^{T} \mathbf{D}+\alpha \mathbf{I}\right)^{-1} \mathbf{D}^{T} \mathbf{y}
\end{aligned}
$$

so it is possible to obtain $\mathbf{x}^{*}$ by simply multiplying $\mathbf{y}$ by a pre-computed matrix $\left(\mathbf{D}^{T} \mathbf{D}+\alpha \mathbf{I}\right)^{-1} \mathbf{D}^{T}$, where $\mathbf{D}$ is from a dataset containing diverse image categories such as Caltech-101 dataset [34] whose images can provide the atoms of a dictionary with sufficiently various shapes. One can use the atoms of a visual dictionary even though they would not be used to classify the images with which they are trained. Thus, the visual dictionary $\mathbf{D}$ does not have to be trained with our experimental videos, and the frame clustering yields reliable results as shown in Fig. 2 that shows the examples of frames belonging to the same cluster. Now that each frame is represented by the coefficient vector defined above, let us denote the center of the vectors in the $q$ th cluster of the $p$ th temporal segment as $\mathbf{m}_{p, q}$. Then, the distance of each frame $f_{i}$ from its center is denoted as $d_{i}$, which is added to the image quality measures. In this situation, each cluster may be either small or big, which would give rise to an unbalanced distance measure. To alleviate this problem, we use Mahalanobis distance to compute $d_{i}$ s.

In summary, the quality measure for $f_{i}$ (the $i$-th frame) is defined as the combination of the distance $d_{i}$, contrast $c_{i}$, blurriness $b_{i}$, and skewness $s_{i}$ in the form of

$$
a_{i}=d_{i} \sqrt{c_{i}+b_{i}+g\left(c_{i}+b_{i}+s_{i}\right)}, \quad i=1, \ldots, n
$$

where $g(x)=x /\left(1+|x|^{l}\right)^{1 / l}(l=10$ in our experiments $)$, and this is also normalized into range of $[0,1] . g(x)$ 

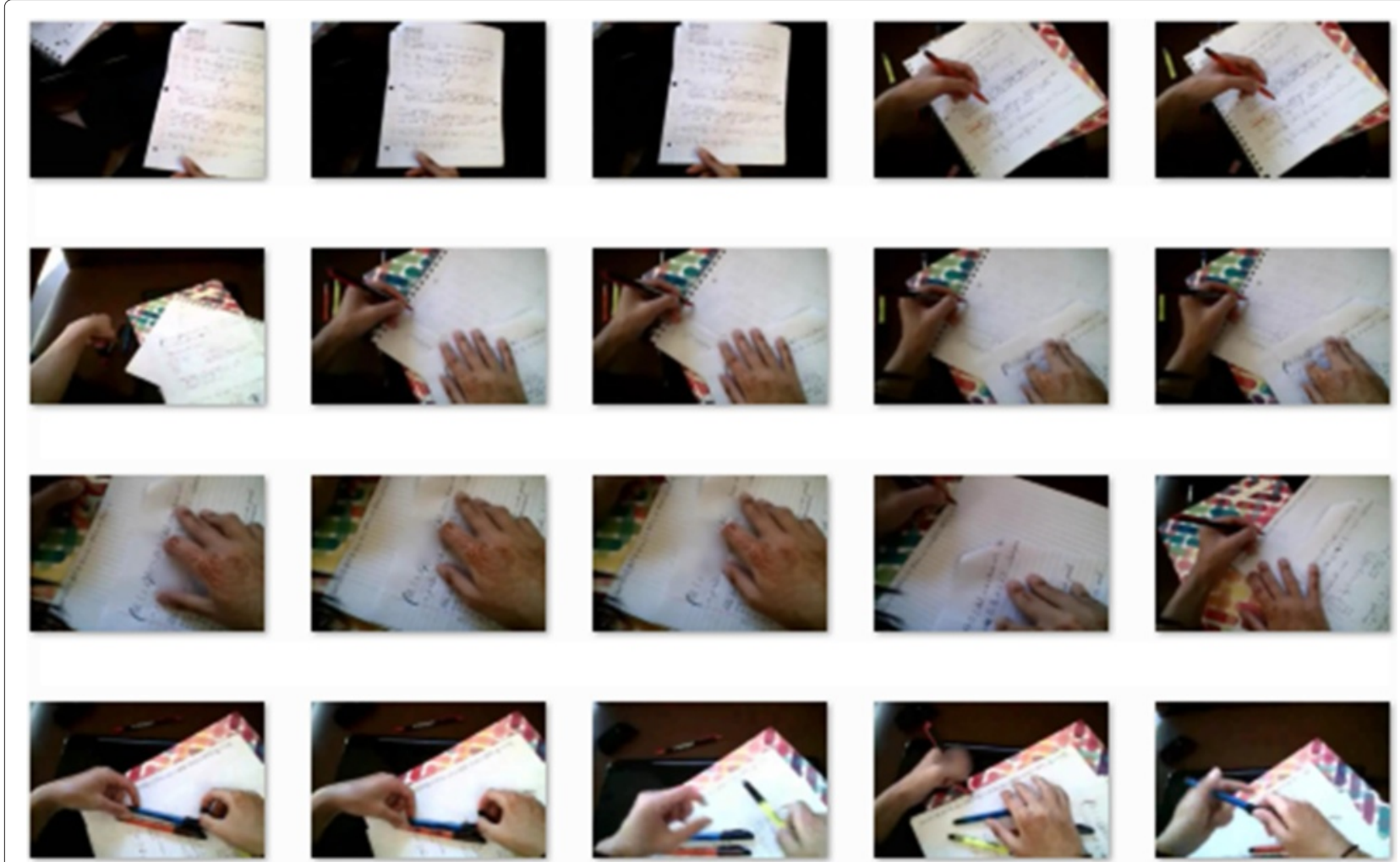

Fig. 2 An example of images belonging to the same cluster. Frames that look similar to each other can agglomerate to form a cluster. The number of its members increases with the time duration of the scene they show

behaves like $g(x)=x$ when $x \in[0,1]$ and like $g(x)=$ 1 when $x \in[1, \infty)$. When $a_{i}$ is close to 0 , then $f_{i}$ is regarded as a good-quality frame. Because skewness does not have any meaning for severely low-contrast or blurred images, it should be used only to find out good frames among high-contrast and non-blurred images. For this reason, we use the $g\left(c_{i}+b_{i}+s_{i}\right)$ term where $s_{i}$ loses its importance when $c_{i}$ or $b_{i}$ is large. Besides, the square root computation is intended to give similar $a_{i}$ values to low-contrast or blurred images, and lastly, $a_{i}$ is multiplied by $d_{i}$ to consider whether or not the scene caught the capturer's attention as mentioned above. Figure 3 shows some frames with the lowest or highest $a_{i}$ values in their clusters to compare these two classes of frames. Finally,

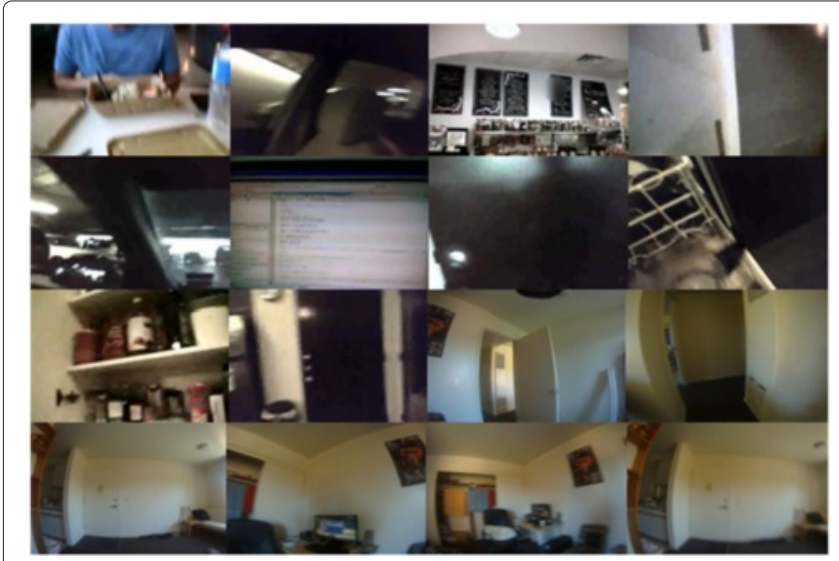

a

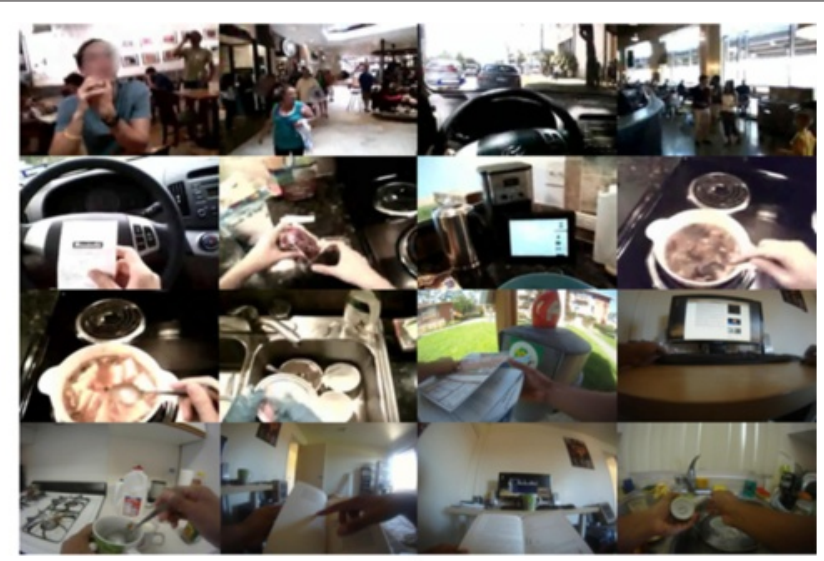

b

Fig. 3 Examples of (a) low-quality and (b) high-quality frames. The frames with the maximum or the minimum cost values in the clusters of some videos are listed. Each image of (b) has good quality itself or shows the stable (or static) scene in its cluster 
$E_{1}(\mathbf{z})$ in (8) is the sum of $a_{i} \mathrm{~s}$ of the frames belonging to a keyframe set.

Based on the clustering result, the representativeness of the keyframes is defined as "how the selected keyframe set is close to the set of cluster centers $\left\{\mathbf{m}_{p, q}\right\}$," which is conceptually defined as

$$
E_{2}(\mathbf{z})=\operatorname{Diff}\left(\mathbf{z},\left\{\mathbf{m}_{p, q}\right\}\right) .
$$

\subsection{Formulation of cost function and its optimization}

With the above conceptual definitions of $E_{1}(\mathbf{z})$ and $E_{2}(\mathbf{z})$, the optimization of (8) is actually formulated as a graph matching problem $[10,11,35]$. Specifically, we define a complete graph $\mathbf{G}=(\mathbf{V}, \mathbf{E})$, where $\mathbf{V}$ is a set of $\mathbf{m}_{p, q} \mathbf{S}$ and every pair of vertices is connected by a single edge. The representativeness of keyframes in (12) is defined as "the similarity of their patterns to those of the cluster centers." For this, suppose that a vector $\mathbf{m}_{p, q}$ can be reconstructed by the linear combination of the other ones. If the number of $\mathbf{m}_{p, q} \mathbf{s}$ is $k$, and we denote the concatenated matrix $\mathbf{P}=\left[\mathbf{m}_{1,1}, \mathbf{m}_{1,2}, \ldots, \mathbf{m}_{p, q}, \ldots\right] \in \mathbb{R}^{d \times k}(d$ is the dimension of $\mathbf{m}_{p, q}$ ), then the coefficients of their linear combinations are given by

$$
\begin{gathered}
\min _{\mathbf{w}_{p, q} \in \mathbb{R}^{k}}\left\|\mathbf{P} \mathbf{w}_{p, q}-\mathbf{m}_{p, q}\right\|_{2}^{2}+\eta\left\|\mathbf{w}_{p, q}\right\|_{2}^{2} \\
\text { s.t. } \mathbf{w}_{p, q}^{T} \mathbf{1}_{k}=1, w_{(p, q), i}=0
\end{gathered}
$$

where $i$ is the index of $\mathbf{m}_{p, q}$ in $\mathbf{P}$. In (13), the first term is for reconstruction errors, and the second is a regularization term that suppresses some big weights and gets $\mathbf{w}_{p, q}$ to prefer to learn small weights. The smaller $\eta$ gets, the more the role of $\mathbf{m}_{p, q}$ 's neighbors is emphasized for its reconstruction. By solving this problem, we can get the concatenated matrix $\mathbf{W}=\left[\mathbf{w}_{1,1}, \mathbf{w}_{1,2}, \ldots, \mathbf{w}_{p, q}, \ldots\right] \in$ $\mathbb{R}^{k \times k}$.

Since each keyframe is extracted from its cluster, let us define a binary association matrix $\mathbf{G} \in\{0,1\}^{k \times n}$ that encodes this, where $G(i, j)=1$ if the $j$ th frame belongs to the $i$ th cluster. In addition, let the cost matrix $\mathbf{A} \in \mathbb{R}^{k \times n}$ contain the overall frame costs, where $A(i, j)=a_{j}$ if $G(i, j)=1$. Also, the feature vectors of all the $n$ frames given by ScSPM form a matrix of the candidates $\mathbf{Q}=$ $\left[\mathbf{q}_{1}, \mathbf{q}_{2}, \mathbf{q}_{3}, \ldots, \mathbf{q}_{n}\right]^{T} \in \mathbb{R}^{n \times d}$. Given the matrices $\mathbf{G}, \mathbf{A}, \mathbf{Q}$, and $\mathbf{W}$, we find the optimal correspondences $\mathbf{X}$ by solving the following problem:

$$
\begin{aligned}
& \min _{\mathbf{z} \subset \mathbf{F}} \lambda E_{1}(\mathbf{z})+E_{2}(\mathbf{z}) \Leftrightarrow \\
& \min _{\mathbf{X}} \lambda \operatorname{trace}\left(\mathbf{A X}^{T}\right)+\left\|\left(\mathbf{I}_{k}-\mathbf{W}\right) \mathbf{X Q}\right\|_{1} \\
& \text { s.t. } \mathbf{X} \in\{0,1\}^{k \times n}, \mathbf{X} \mathbf{1}_{n}=\mathbf{1}_{k}, \\
& \quad X(i, j)=0 \text { if } G(i, j)=0
\end{aligned}
$$

where $\lambda$ is a regularization weight for the trade-off between the frame cost and the reconstruction error.
Finding $\mathbf{X}$ is to get the keyframe set $\mathbf{z}^{*} \subset \mathbf{F}$, so $E_{1}(\mathbf{z})$ and $E_{2}(\mathbf{z})$ correspond to trace $\left(\mathbf{A} \mathbf{X}^{T}\right)$ and $\left\|\left(\mathbf{I}_{k}-\mathbf{W}\right) \mathbf{X Q}\right\|_{1}$ respectively where the former means the sum of $a_{i} \mathrm{~s}$ of the selected keyframes and the latter decreases as the patterns of the keyframe set and $\mathbf{P}$ get similar to each other. Multiplying a frame cost by $d_{i}$ in (11) allows this optimization problem to be stable, which means that it reduces the influence of $\lambda$ 's variation. However, the problem of (14) is NP-hard and nonlinear with integer constraints. To efficiently solve this problem, the reconstruction error term is linearized and the binary constraint $\mathbf{X} \in\{0,1\}^{k \times n}$ is relaxed to the continuous domain $[0,1]^{k \times n}$, leading to the following problem:

$$
\begin{aligned}
& \min _{\mathbf{z} \subset \mathbf{F}} \lambda E_{1}(\mathbf{z})+E_{2}(\mathbf{z}) \Leftrightarrow \\
& \min _{X, U, V} \lambda \operatorname{trace}\left(\mathbf{A X}^{T}\right)+\mathbf{1}_{k}^{T}(\mathbf{U}+\mathbf{V}) \mathbf{1}_{d} \\
& \text { s.t. } \mathbf{U} \geq \mathbf{0}_{k \times d}, \mathbf{V} \geq \mathbf{0}_{k \times d},\left(\mathbf{I}_{k}-\mathbf{W}\right) \mathbf{X Q}=\mathbf{U}-\mathbf{V} \\
& \quad \mathbf{X} \in[0,1]^{k \times n}, \mathbf{X} \mathbf{1}_{n}=\mathbf{1}_{k}, \\
& \quad X(i, j)=0 \text { if } G(i, j)=0
\end{aligned}
$$

where $\mathbf{U}, \mathbf{V} \in \mathbb{R}^{k \times d}$ are the auxiliary matrices representing upper- and lower-bounds respectively for $\left\|\left(\mathbf{I}_{k}-\mathbf{W}\right) \mathbf{X Q}\right\|_{1}$, so $\mathbf{U}+\mathbf{V}$ means the size of the interval for $\left(\mathbf{I}_{k}-\mathbf{W}\right) \mathbf{X Q}$, the norm of which is minimized along with $\mathbf{1}_{k}^{T}(\mathbf{U}+\mathbf{V}) \mathbf{1}_{d}$. This problem can be solved with linear programming. Last, to discretize $\mathbf{X}$, we perform the iterated conditional modes (ICM) row by row for every entry of $\mathbf{X}$. As a result of the above procedure, $\mathbf{X}$ has only $k 1$ s. Hence, if the $i$ th column of $\mathbf{X}$ has a non-zero entry, then it means that $f_{i}$ is selected as a keyframe. After sorting by time, we obtain the final storyboard. Finally, the overview of our method is illustrated in Fig. 4.

\section{Experimental results}

In our experiments, the UTE (UT Egocentric) [2] and the ADL (activities of daily living) [36] databases were used. The UTE dataset consists of 4 videos that are about 3 $5 \mathrm{~h}$ long, and the ADL dataset contains 20 videos, most of which are less than an hour long. For the comparison, we selected 5 different baselines: (1) uniform sampling, (2) clustering-based (with color histograms) [4], (3) visual attention-based [8], (4) object-driven (only for the UTE dataset) [2], and (5) our method without the optimization procedure. What we intend by the last one is that the frame that has the lowest cost in each cluster is selected as a keyframe.

Several parameters are involved in our algorithm: $\theta_{a}, \theta_{b}$ for spectral clustering, $C$ in the KTS step, $\alpha$ in the feature extraction step, and $\eta, \lambda$ in the optimization step. We set $\theta_{a}=10^{-3}, \alpha=0.15, \eta=10^{-1}$ in all the experiments, and $\theta_{b} \in[0.05,0.15], C \in[1,3]$ where we 


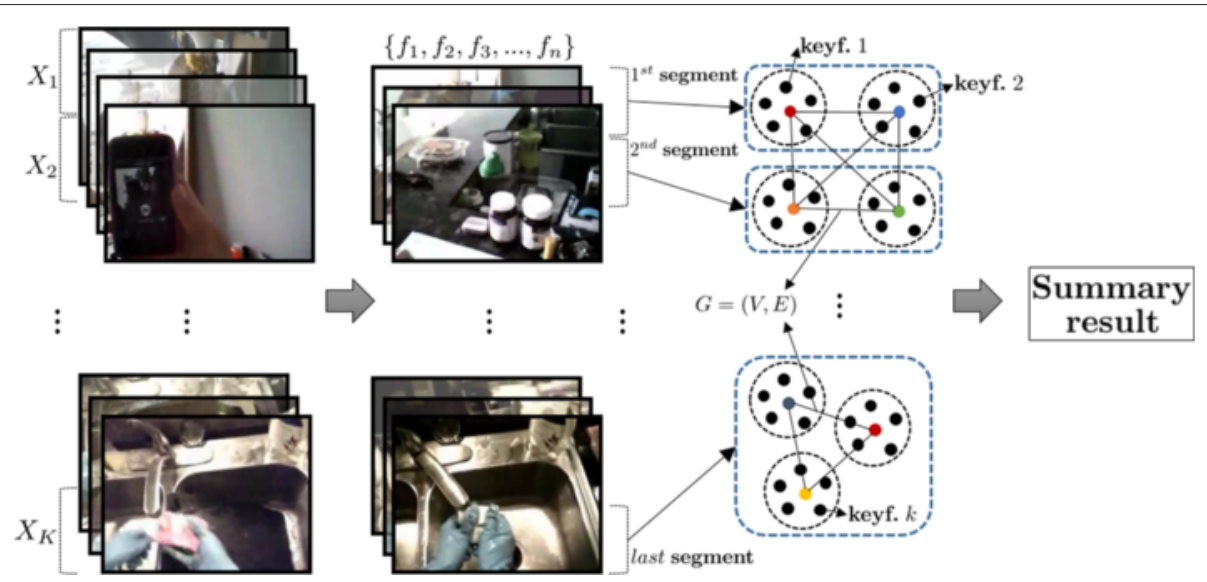

Fig. 4 Overview of our method. $\left\{f_{1}, f_{2}, f_{3}, \ldots, f_{n}\right\}$ is the set of remaining frames after redundant frame pruning, and $m_{p, q} s$ combine to form a fiducial graph described in this figure. $k$ frames are selected as the keyframes and sorted by time to result in the final summary

controlled them depending on the length of a video. Also, the value of $\lambda$ was adjusted between $10^{1}$ and $10^{3}$. The value of $\eta$ can be determined by examining the reconstruction error in (14), and it could be set as the value with the minimum reconstruction error for each input video; but we used the value of $\eta$ consistent in every experiment for simplicity-to avoid repeating the same steps.

It has often been seen in the literature that only meanopinion scores (MOS) are used for evaluation, while some researchers recently exploited the precision and recall criterion $[1,8]$ for objectiveness, especially for static summarization. For this, a human subject makes his/her own summary and then it is compared to the summaries made by automatic methods. Because comparing two images is itself subjective, it is important to reduce the subjectivity in this work. Hence, we asked 11 subjects to take the following steps with the videos of the two datasets in the experiments: (1) summarize a video with a series of events in words, (2) label the keyframes corresponding to the events in an automatic summarization result, and lastly (3) score each video summary from 0 to 5 . We made every subject sure that an event may be matched to nothing or to more than one keyframe. Given the questionnaires submitted by the subjects, we counted the number of labeled keyframes $\left(N_{L K}\right)$ and events reflected in a summary $\left(N_{R E}\right)$ to compute precision and recall scores. These are given by

$$
\begin{gathered}
\text { Precision }=\frac{N_{L K}}{N_{K}}, \quad \text { Recall }=\frac{N_{R E}}{N_{E}} \\
F \text {-measure }=2 \frac{\text { Precision } \times \text { Recall }}{\text { Precision }+ \text { Recall }}
\end{gathered}
$$

where $N_{K}$ and $N_{E}$ are the numbers of keyframes and events respectively. Tables 1 and 2 show these quantitative results on the UTE and the ADL datasets, respectively. The clustering-based and the visual attention-based methods shared the temporal segmentation results with ours, but the latter one performed temporal segmentation once more on each video segment to get more and shorter segments so that one frame with the highest score could be selected in each segment. The methods used to compare with each other resulted in the similar number of keyframes in our experiments, and we believe that it is the appropriate way to make both the best results of each method and a fair comparison. Some examples of video summaries are shown in Figs. 5 and 6.

The MOS criterion reflects the overall quality of a summary, particularly regarding image quality, important objects, and so on. Because each subject has his/her own scoring criterion, we used the standard scores (i.e., score $\leftarrow 5+1 \cdot$ (score - mean)/std_dev) and confidence intervals. Table 3 shows the MOSs, and it means that the general criteria on keyframe quality (i.e., contrast, blurriness, skewness, and duration of its scene) are efficient for video summarization. These criteria do not require much computational load and can be applied to videos with various lengths. The MOS results also show that the proposed method attained better scores than the other ones, as the precision-recall results do.

It can be seen with the quantitative results that the clustering-based algorithm produce better summaries for the ADL dataset than for the UTE dataset, and we infer that it is because the videos in the ADL dataset have large variations in color, and the scenes with different color distributions tend to correspond to different activities. Since the clustering-based method depends only on color histogram features, it may have trouble finding out a meaningful frame from a scene with consistently colored frames. In Fig. 5, the fifth image in the second row 
Table 1 UTE dataset results averaged w.r.t. subjects

\begin{tabular}{|c|c|c|c|c|c|c|c|c|}
\hline Method & Video name & $N_{R E}$ & $N_{E}$ & $N_{L K}$ & $N_{K}$ & Precision & Recall & $F$-measure \\
\hline \multirow[t]{5}{*}{ Uniform sampling } & P01 & 6.3 & 9.5 & 7.6 & 12 & 0.636 & 0.711 & 0.656 \\
\hline & P02 & 7.8 & 10.2 & 8.9 & 15 & 0.594 & 0.778 & 0.668 \\
\hline & P03 & 4.5 & 8.3 & 5.9 & 12 & 0.492 & 0.545 & 0.513 \\
\hline & P04 & 6.4 & 9.0 & 7.6 & 16 & 0.477 & 0.718 & 0.565 \\
\hline & Avg. & 6.2 & 9.3 & 7.5 & 13.8 & 0.550 & 0.688 & 0.600 \\
\hline \multirow[t]{5}{*}{ Clustering-based [4] } & P01 & 6.7 & 9.5 & 7.6 & 11 & 0.694 & 0.755 & 0.709 \\
\hline & P02 & 8.4 & 10.2 & 10.5 & 16 & 0.653 & 0.823 & 0.720 \\
\hline & P03 & 5.5 & 8.3 & 7.5 & 14 & 0.532 & 0.664 & 0.588 \\
\hline & P04 & 7.9 & 9.0 & 10.1 & 18 & 0.561 & 0.894 & 0.677 \\
\hline & Avg. & 7.1 & 9.3 & 8.9 & 14.8 & 0.610 & 0.784 & 0.674 \\
\hline \multirow[t]{5}{*}{ Attention-based [2] } & P01 & 7.1 & 9.5 & 7.9 & 12 & 0.659 & 0.790 & 0.708 \\
\hline & P02 & 6.0 & 10.2 & 6.8 & 13 & 0.524 & 0.601 & 0.555 \\
\hline & P03 & 5.5 & 8.3 & 7.0 & 12 & 0.583 & 0.661 & 0.611 \\
\hline & P04 & 7.3 & 9.0 & 8.5 & 16 & 0.534 & 0.811 & 0.634 \\
\hline & Avg. & 6.5 & 9.3 & 7.6 & 13.3 & 0.575 & 0.716 & 0.627 \\
\hline \multirow[t]{5}{*}{ Object-driven [8] } & P01 & 7.0 & 9.5 & 9.4 & 13 & 0.720 & 0.776 & 0.731 \\
\hline & P02 & 7.5 & 10.2 & 10.9 & 19 & 0.574 & 0.741 & 0.641 \\
\hline & P03 & 6.0 & 8.3 & 8.2 & 12 & 0.682 & 0.720 & 0.692 \\
\hline & P04 & 7.0 & 9.0 & 8.5 & 16 & 0.534 & 0.793 & 0.632 \\
\hline & Avg. & 6.9 & 9.3 & 9.3 & 15.0 & 0.628 & 0.758 & 0.674 \\
\hline \multirow[t]{5}{*}{ Proposed (w/o optimization) } & P01 & 6.1 & 9.5 & 6.5 & 10 & 0.655 & 0.686 & 0.659 \\
\hline & P02 & 7.1 & 10.2 & 8.1 & 13 & 0.622 & 0.704 & 0.655 \\
\hline & P03 & 5.8 & 8.3 & 7.4 & 11 & 0.669 & 0.707 & 0.683 \\
\hline & P04 & 7.7 & 9.0 & 8.8 & 15 & 0.588 & 0.867 & 0.689 \\
\hline & Avg. & 6.7 & 9.3 & 7.7 & 12.3 & 0.634 & 0.741 & 0.672 \\
\hline \multirow[t]{5}{*}{ Proposed } & P01 & 7.1 & 9.5 & 7.8 & 10 & 0.782 & 0.791 & 0.773 \\
\hline & P02 & 8.2 & 10.2 & 9.3 & 13 & 0.713 & 0.811 & 0.756 \\
\hline & P03 & 6.8 & 8.3 & 8.5 & 11 & 0.777 & 0.830 & 0.798 \\
\hline & P04 & 7.9 & 9.0 & 9.5 & 15 & 0.630 & 0.889 & 0.725 \\
\hline & Avg. & 7.5 & 9.3 & 8.8 & 12.3 & 0.726 & 0.830 & 0.763 \\
\hline
\end{tabular}

of this method's result is an example that shows this case very well. It represents the scene at a kitchen, but it fails to show us what the capturer is mainly doing there. Using only color features also has the limit that it has trouble detecting a shot boundary between two video shots similar in color [3]. Although the attention-based method extracts good keyframes in static scenes, it also selects the frames with strong motions as keyframes as shown in Fig. 6, which shows many transient scenes where the capturer is moving. It made us adjust the weight between

Table 2 ADL dataset results averaged w.r.t. subjects and videos

\begin{tabular}{|c|c|c|c|c|c|c|c|}
\hline Method & $N_{R E}$ & $N_{E}$ & $N_{L K}$ & $N_{K}$ & Precision & Recall & $F$-measure \\
\hline Uniform sampling & 5.5 & 8.3 & 6.2 & 9.2 & 0.685 & 0.705 & 0.685 \\
\hline Clustering-based [4] & 6.4 & 8.3 & 6.8 & 8.8 & 0.778 & 0.789 & 0.772 \\
\hline Attention-based [8] & 5.8 & 8.3 & 6.6 & 9.5 & 0.705 & 0.746 & 0.710 \\
\hline Proposed (w/o optimization) & 6.3 & 8.3 & 7.0 & 9.2 & 0.779 & 0.784 & 0.769 \\
\hline Proposed & 6.6 & 8.3 & 7.3 & 9.2 & 0.810 & 0.803 & 0.793 \\
\hline
\end{tabular}



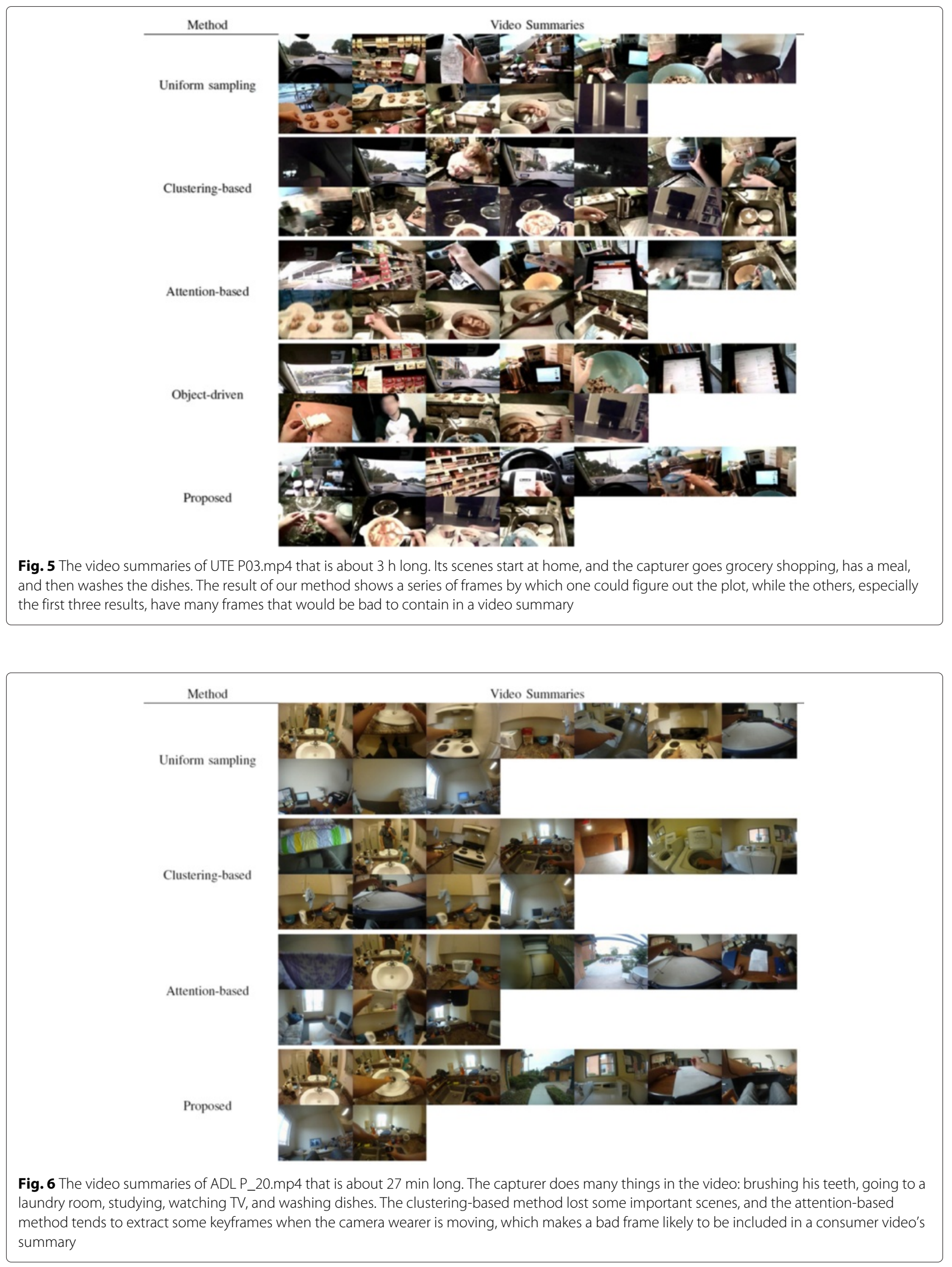
Table 3 Average standard MOS results with $95 \%$ confidence intervals in our experiments

\begin{tabular}{clc}
\hline Dataset & Method & MOS \\
\hline UTE & Uniform sampling & $4.17 \pm .30$ \\
& Clustering-based [4] & $5.10 \pm .29$ \\
& Attention-based [8] & $4.49 \pm .23$ \\
& Object-driven [2] & $5.36 \pm .32$ \\
& Proposed (w/o optimization) & $5.18 \pm .22$ \\
& Proposed & $5.69 \pm .29$ \\
ADL & Uniform sampling & $4.41 \pm .20$ \\
& Clustering-based [4] & $5.25 \pm .18$ \\
& Attention-based [8] & $4.57 \pm .16$ \\
& Proposed (w/o optimization) & $5.26 \pm .14$ \\
& Proposed & $5.51 \pm .16$ \\
\hline
\end{tabular}

the spatial and the temporal attention values described in [8] to obtain better results with the longer and more complicated videos. For this reason, the attention-based method seems to be more suitable for edited videos or those with lesser motions. The object-driven method has the superior abilities to find out keyframes with good quality of frames themselves, but it may give rise to false positives in a scene. If a scene shows many conspicuous things such as objects, hands, and faces, the algorithm tends to extract more keyframes in that scene than in others, which may make a subject regard them as false positives. For example, this method's result in Fig. 5 has many keyframes-in a certain sense, too many as compared to the video's length - that show the scene at the kitchen and the fifth and sixth keyframes are almost the same. As a results, the object-driven method attained better results in evaluation on MOS scores than on precision-recall scores. The methods using clustering such as the clustering-based one and ours have a problem with the number of clusters, because it determines the number of keyframes and whether or not some scene is reflected in a video summary. The first two frames of our result in Fig. 6 show the same activity, so some of the subjects regarded one of them as a redundant keyframe. Also, the first keyframe of our result in Fig. 5 may be regarded as a false positive because the capturer stays home for a short time at the beginning of this video. Figure 7 shows a worse case with such problem. These redundant keyframes would disappear with a shorter summary's length setting, but it might cause other proper keyframes to also be removed because of clustering with a constant threshold.

As mentioned above, we are given the choice between choosing representative frames and quality ones, so we formulate the last step in our algorithm as an optimization problem. Figure 8 shows the frames with the minimum values of $a_{i}$ or $d_{i}$ in their clusters and the keyframes resulted from our optimization step with various values of $\lambda$. This figure confirms that the keyframes with a small $\lambda$ value are similar to those closest to the centers of their clusters, while the ones with a larger $\lambda$ value tend to be similar to those with the minimum $a_{i}$ values, as expected. In our experiments, the frames at the second row in Fig. 8 had little differences in the values of $a_{i}$ and $d_{i}$ with one another, so the keyframe results did not have exactly the same frames with the minimum $a_{i}$ or $d_{i}$ values in that cluster. While any image may be qualified as a good keyframe at the second row, the summary that has a laundry room image as the first keyframe is more suitable in terms of the video's story, which led to the higher scores on both the precision and the recall criteria. However, someone might prefer the first frame at the first row, and this is basically a matter of opinion.

We conducted additional experiments to confirm how our method deals with the videos showing camerawork such as camera pan and zoom. The SumMe dataset [1] contains both first-person and third-person videos, which

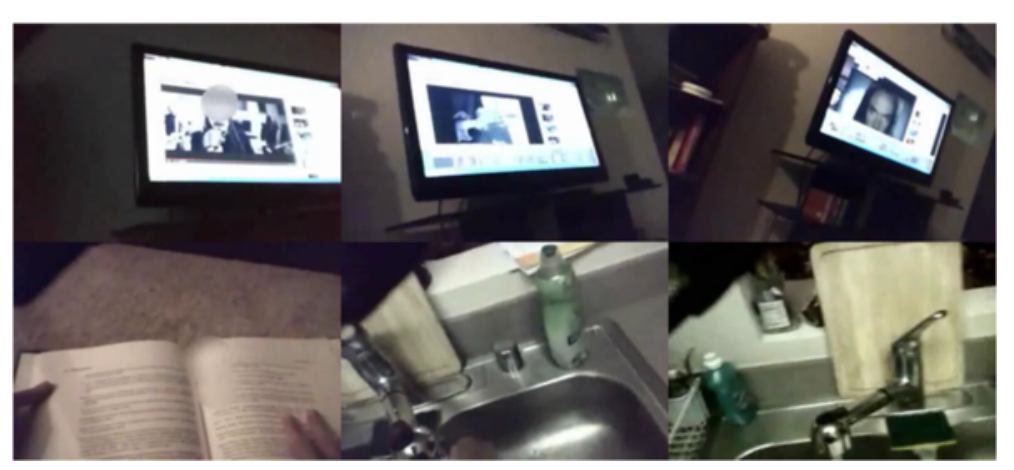

Fig. 7 A part of our summary on UTE P_02.mp4 showing the problem with the number of clusters. Clustering with a constant threshold may produce several clusters similar to each other. Most of the subjects regarded roughly half of the keyframes in this part as of little importance 


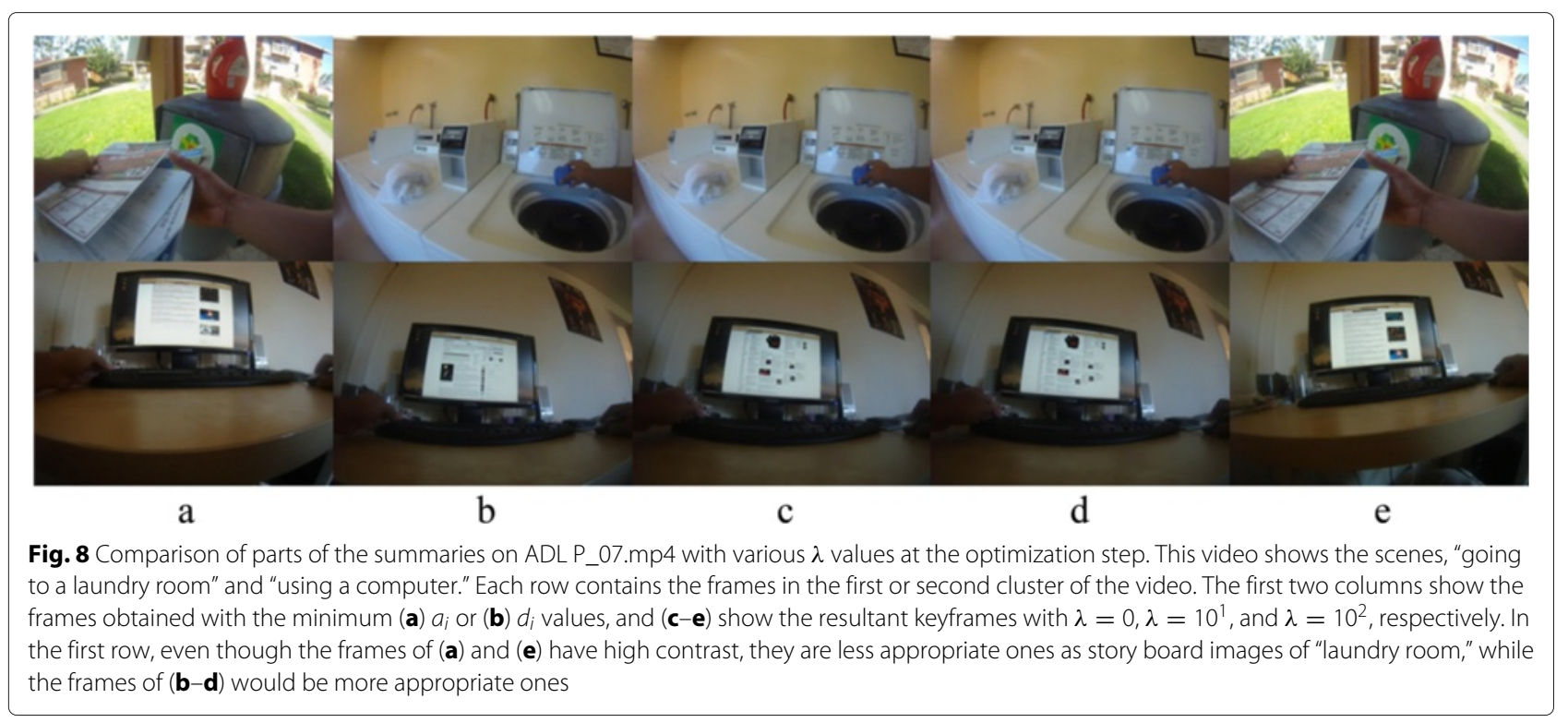

are a few minutes long, so we summarized the thirdperson videos with strong camerawork as shown in Fig. 9. A scene with camera zoom probably has important information (e.g., prominent objects), and if so, one of the frames within the scene qualifies for a keyframe. Those scenes are usually long or stable enough to clearly show important information, which can be encoded by our representativeness measure. The keyframes with red borders in Fig. 9 were captured at the moments of zoom, and they hold a majority in the summaries. On the one hand, a scene with camera pan is probably transient and not suitable to have a keyframe; in fact, the video skimming and keyframe selection steps are likely to preclude those scenes from a summary. However, they sometimes have stable and meaningful information when the camera keeps tracking some objects as the third image in Fig. 9a. Even though this scene was captured at the moment of pan and is rather short, many frames that look similar to each other formed a cluster and yielded a keyframe. Measuring duration of a scene can help to decide whether or not a scene with zoom or pan deserves to be included in a summary, and the duration is one of our criteria for selecting keyframes.

As shown in Figs. 5 and 6, the summaries are different in length, which is because all but the uniform sampling method cannot set the exact number of keyframes in the experiments. This may be a limitation of our algorithm. Finally, as stated previously, some of the skimmed videos and static summary results are shown in http://ispl.snu.ac. $\mathrm{kr} / \sim$ jeongdj/videosummary.html.

\section{Conclusions}

We have proposed a video summarization algorithm for consumer videos, which is specifically designed for the

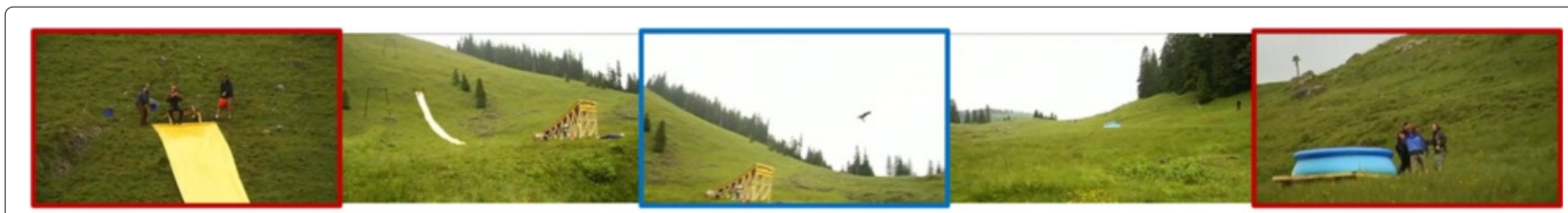

a

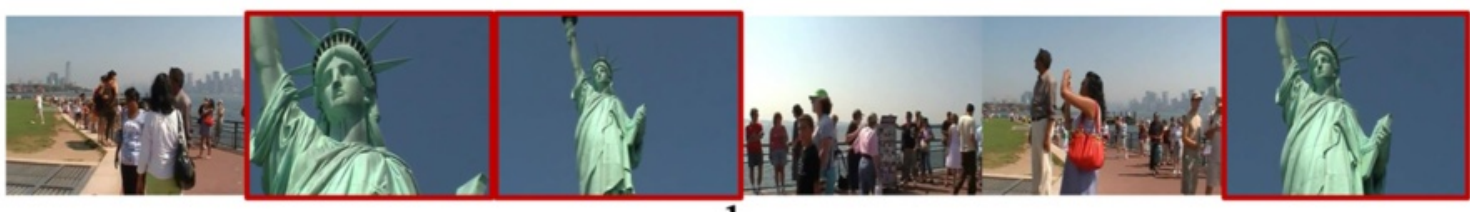

b

Fig. 9 The summaries of the videos with strong camerawork. a Jumps.mp4 and (b) Statue of Liberty.mp4 in the SumMe dataset show consistent camerawork such as zoom and pan. Red and blue borders indicate that their frames were captured at the moments of zoom and pan, respectively 
long and unedited videos. For the fast processing, the video is first roughly segmented and clustered to be a skimmed video, and then the keyframes with good quality and representativeness are selected. At the first video skimming step, our method removes most of the redundant frames very fast by applying spectral clustering on the evenly spaced segments with simple color histogram features. In the second keyframe selection step, we formulate the keyframe extraction as an optimization problem, where we consider the representativeness as the effectiveness of being a linear combination of other keyframes, and also consider quality in terms of contrast, blurriness, skewness, and distinctiveness. Experimental results show that the proposed method works well on the UTE and the ADL datasets but shows some limitation in that the number of keyframes is not finely controlled, which needs further research in the clustering and optimization processes.

\section{Endnote}

${ }^{1}$ The notations related to the spectral clustering are only for this section.

\section{Competing interests}

The authors declare that they have no competing interests.

\section{Authors' contributions}

- A video summarization method is proposed, which is specifically suited for long and unedited consumer videos.

- For the fast summary of long and unedited videos, the input video is first skimmed by spectral clustering with simple color histogram features.

- We balance the representativeness and image quality when selecting keyframes, because there are many bad quality images in consumer videos, which is formulated as an optimization problem over a graph.

- The representativeness is formulated in terms of similarity to cluster centers, so that the selected frames effectively cover many of similar frames in a subshot.

\section{Acknowledgements}

This research was supported by Projects for Research and Development of Police Science and Technology under Center for Research and Development of Police science and Technology and Korean National Police Agency (PA-C000001).

\section{Author details}

${ }^{1}$ Department of Electrical and Computer Engineering, INMC, Seoul National University, 1 Gwanak-ro, Gwanak-gu, Seoul 08826, Korea. ${ }^{2}$ Samsung Electronics, Ltd., 426-910 Gyeonggi Do, Suwon, Korea.

Received: 9 November 2015 Accepted: 12 June 2016

Published online: 22 June 2016

\section{References}

1. M Gygli, H Grabner, H Riemenschneider, L Van Gool, in European Conference on Computer Vision. Creating summaries from user videos, vol. 8695, (2014), pp. 505-520

2. YJ Lee, J Ghosh, K Grauman, in IEEE Conference on Computer Vision and Pattern Recognition. Discovering important people and objects for egocentric video summarization, (2012), pp. 1346-1353

3. M Omidyeganeh, S Ghaemmaghami, S Shirmohammadi, Video keyframe analysis using a segment-based statistical metric in a visually sensitive parametric space. IEEE Trans Image Process. 20(10), 2730-2737 (2011)

4. VT Chasanis, CL Likas, NP Galatsanos, Scene detection in videos using shot clustering and sequence alignment. IEEE Trans. Multimed. 11(1), 89-100 (2009)
5. SEF de Avila, APB Lopes, A da Luz Jr, A de Albuquerque Araújo, Vsumm: a mechanism designed to produce static video summaries and a nove evaluation method. Pattern Recogn Lett. 32(1), 56-68 (2011)

6. BT Truong, S Venkatesh, Video abstraction: a systematic review and classification. ACM Trans Multimedia Comput Commun Appl. 3(1) (2007). doi:10.1145/1198302.1198305

7. Y Cong, J Yuan, J Luo, Towards scalable summarization of consumer videos via sparse dictionary selection. IEEE Trans Multimed. 14(1), 66-75 (2012)

8. N Ejaz, I Mehmood, SW Baik, Efficient visual attention based framework for extracting key frames from videos. Signal Process Image Commun. 28(1), 34-44 (2013)

9. Z Lu, K Grauman, in IEEE Conference on Computer Vision and Pattern Recognition. Story-driven summarization for egocentric video, (2013), pp. 2714-2721

10. H Jiang, M Drew, Z-N Li, Matching by linear programming and successive convexification. IEEE Trans Pattern Anal Mach Intell. 29(6), 959-975 (2007)

11. H Li, X Huang, L He, Object matching using a locally affine invariant and linear programming techniques. IEEE Trans Pattern Anal Mach Intell. 35(2), 411-424 (2013)

12. M Furini, F Geraci, M Montangero, M Pellegrini, Stimo: still and moving video storyboard for the web scenario. Multimedia Tools Appl. 46(1), 47-69 (2010)

13. N Ejaz, TB Tariq, SW Baik, Adaptive key frame extraction for video summarization using an aggregation mechanism. J Vis Commun Image Represent. 23(7), 1031-1040 (2012)

14. W Chen, YJ Zhang, Parametric model for video content analysis. Pattern Recogn Lett. 29(3), 181-191 (2008)

15. M Cooper, J Foote, in IEEE International Conference on Multimedia and Expo. Discriminative techniques for keyframe selection, (2005), pp. 502-505

16. A Amiri, M Fathy, Hierarchical keyframe-based video summarization using QR-decomposition and modified k-means clustering. EURASIP J Adv Signal Process. 2010:892124, 1-16 (2010)

17. M Guironnet, D Pellerin, N Guyader, P Ladret, Video summarization based on camera motion and a subjective evaluation method. EURASIP J Image Video Process. 2007:060245, 1-12 (2007)

18. GG Lakshmi Priya, S Domnic, Walsh-hadamard transform kernel-based feature vector for shot boundary detection. IEEE Trans Image Process. 23(12), 5187-5197 (2014)

19. P Mohanta, S Saha, B Chanda, A model-based shot boundary detection technique using frame transition parameters. IEEE Trans Multimed. 14(1), 223-233 (2012)

20. M Birinci, S Kiranyaz, A perceptual scheme for fully automatic video shot boundary detection. Signal Process Image Commun. 29(3), 410-423 (2014)

21. D Potapov, M Douze, Z Harchaoui, C Schmid, in European Conference on Computer Vision. Category-specific video summarization, vol. 8694, (2014), pp. 540-555

22. Y Poleg, C Arora, S Peleg, in IEEE Conference on Computer Vision and Pattern Recognition. Temporal segmentation of egocentric videos, (2014), pp. 1346-1353

23. I González-Díaz, T Martínez-Cortés, A Gallardo-Antolín, F Díaz-de-María, Temporal segmentation and keyframe selection methods for user-generated video search-based annotation. Expert Syst Appl. 42(1) 488-502 (2015)

24. G Abdollahian, CM Taskiran, Z Pizlo, EJ Delp, Camera motion-based analysis of user generated video. IEEE Trans Multimed. 12(1), 28-41 (2010)

25. AY Ng, Ml Jordan, Y Weiss, in Advances in Neural Information Processing Systems. On spectral clustering: Analysis and an algorithm, (2001), pp. 849-856

26. R Arandjelovic, A Zisserman, in IEEE Conference on Computer Vision and Pattern Recognition. Three things everyone should know to improve object retrieval, (2012), pp. 2911-2918

27. H Zha, X He, CHQ Ding, M Gu, HD Simon, in Advances in Neural Information Processing Systems. Spectral relaxation for k-means clustering, (2001), pp. 1057-1064

28. A Likas, N Vlassis, JJ Verbeek, The global k-means clustering algorithm Pattern Recognit. 36(2), 451-461 (2003)

29. F Crete, T Dolmiere, P Ladret, M Nicolas, The blur effect: perception and estimation with a new no-reference perceptual blur metric. Proc SPIE. 6492, 64920-6492011 (2007) 
30. HI Koo, $\mathrm{NI}$ Cho, Skew estimation of natural images based on a salient line detector. J Electron Imaging. 22(1), 013020-013020 (2013)

31. J Yang, $\mathrm{K} Y \mathrm{Yu}, \mathrm{Y}$ Gong, $\mathrm{T}$ Huang, in IEEE Conference on Computer Vision and Pattern Recognition. Linear spatial pyramid matching using sparse coding for image classification, (2009), pp. 1794-1801

32. D Zhang, M Yang, X Feng, in IEEE International Conference on Computer Vision. Sparse representation or collaborative representation: which helps face recognition? (2011), pp. 471-478

33. J Waqas, Z Yi, L Zhang, Collaborative neighbor representation based classification using $l 2$-minimization approach. Pattern Recogn Lett. 34(2), 201-208 (2013)

34. L Fei-Fei, R Fergus, P Perona, Learning generative visual models from few training examples: an incremental bayesian approach tested on 101 object categories. Computer Vission Image Understading. 106(1), 59-70 (2007)

35. F Zhou, J Brandt, Z Lin, in IEEE International Conference on Computer Vision. Exemplar-based graph matching for robust facial landmark localization, (2013), pp. 1025-1032

36. H Pirsiavash, D Ramanan, in IEEE Conference on Computer Vision and Pattern Recognition. Detecting activities of daily living in first-person camera views, (2012), pp. 2847-2854

\section{Submit your manuscript to a SpringerOpen ${ }^{\circ}$ journal and benefit from:}

- Convenient online submission

- Rigorous peer review

- Immediate publication on acceptance

- Open access: articles freely available online

- High visibility within the field

- Retaining the copyright to your article

Submit your next manuscript at $\gg$ springeropen.com 